\title{
Determinants of Intracranial Hemorrhage Occurrence and Outcome after Neurothrombectomy Therapy: Insights from the Solitaire FR With Intention For Thrombectomy Randomized Trial
}

\author{
R. Raychev, R. Jahan, DD. Liebeskind, W. Clark, DR.G. Nogueira, and J. Saver; The SWIFT Trial Investigators
}

\begin{abstract}
BACKGROUND AND PURPOSE: Intracranial hemorrhage is the most dreaded complication of neurothrombectomy therapy for acute ischemic stroke. The determinants of intracranial hemorrhage and its impact on clinical course remain incompletely delineated. The purpose of this study is to further investigate the clinical and procedural factors leading to intracranial hemorrhage and to define the clinical impact of different hemorrhagic subtypes.
\end{abstract}

MATERIALS AND METHODS: We analyzed data prospectively collected in the Solitaire FR With Intention for Thrombectomy randomized clinical trial. A multivariable logistic regression model was used to identify independent clinical, imaging, and procedural predictors of any intracranial hemorrhage and of 7 intracranial hemorrhage subtypes. Univariate analysis was used to determine the impact of each of the intracranial hemorrhage subtypes on clinical outcome.

RESULTS: Among the 144 enrolled patients, any radiologic intracranial hemorrhage (21.3\% versus $38.2 \%, P=.035)$, symptomatic intracranial hemorrhage (1.1\% versus $10.9 \%, P=.012$ ), and subarachnoid hemorrhage ( $2.2 \%$ versus $12.7 \%, P=.027$ ) occurred less frequently in the Solitaire FR than in the Merci retriever arms. The most common independent determinant of hemorrhage occurrence was rescue therapy with intra-arterial rtPA, which was associated with any intracranial hemorrhage and 4 subtypes and tended to be used more frequently in the Merci group (10.9\% versus 3.4\%; $P=.09)$. Among the hemorrhage subtypes, basal ganglionic hemorrhage had the strongest impact on good clinical outcome at 90 days (OR, $0.30 ; P=.025)$ and was associated with higher reperfusion, prolonged time to treatment, and rescue therapy with intra-arterial rtPA.

CONCLUSIONS: Intracranial hemorrhage, especially subarachnoid and symptomatic intracerebral hemorrhage, occurs less frequently with the Solitaire FR than the Merci retriever, in part due to less frequent use of rescue therapy with intra-arterial rtPA. Basal ganglionic hemorrhage strongly affects clinical outcome and is distinctively related to late reperfusion.

ABBREVIATIONS: IA = intra-arterial; ICH = intracranial hemorrhage; IVH = intraventricular hemorrhage; $\mathrm{PH}=$ parenchymal hematoma; $\mathrm{SICH}=$ symptomatic intracerebral hemorrhage; SWIFT = Solitaire FR With Intention For Thrombectomy

S ince the introduction of the recanalization therapy for acute ischemic stroke, both via intravenous fibrinolysis and catheter-based reperfusion, intracranial hemorrhage ( $\mathrm{ICH}$ ) has remained the most feared complication. The potential occurrence of ICH has required that the main risk of acute revascularization be balanced against the benefit of reperfusion. ${ }^{1}$ Various pretreat-

Received January 21, 2015; accepted after revision April 16

From the Department of Neurology (R.R., D.L., J.S.), Division of Interventional Neuroradiology (R.J.), and Stroke Center (R.R., R.J., D.L., J.S.), University of California, Los Angeles, Los Angeles, California; Oregon Stroke Center (W.C.), Oregon Health and Sciences University, Portland, Oregon; and Department of Neurology (R.G.N.), Emory University, Atlanta, Georgia.

Please address correspondence to Radoslav Raychev, MD, UCLA Stroke Center, 710 Westwood Plaza RNRC, Los Angeles, CA 90095; e-mail: rudoray@gmail.com; rraychev@mednet.ucla.edu

Evidence-Based Medicine Level 2.

http://dx.doi.org/10.3174/ajnr.A4482 ment clinical and imaging factors have been associated with hemorrhagic transformation after reperfusion treatment, including age, severity of pretreatment neurologic deficit, ischemic lesion volume, elevated blood pressure, serum glucose level, history of diabetes, and absence of collateral flow. ${ }^{2-5}$ However, most available data on $\mathrm{ICH}$ following recanalization therapy in acute ischemic stroke are from studies of intravenous and intra-arterial pharmacologic fibrinolysis and little is known about the clinical and procedural factors contributing to $\mathrm{ICH}$ occurrence after mechanical neurothrombectomy. ${ }^{6,7}$

The clinical impact of different ICH subtypes has also been incompletely studied. Initial emphasis in the intravenous lytic literature focused on symptomatic intracerebral hemorrhage (SICH), referring to any intracranial hemorrhage associated with early neurologic deterioration. However, multiple contending criteria have been advanced for defining an intracerebral hemor- 
Table 1: Factors included in the prediction model

\begin{tabular}{ll}
\hline \multicolumn{1}{c}{ Clinical } & \multicolumn{1}{c}{ Procedural } \\
\hline Age (yr) & Vessel treated (ICA/carotid-T vs MI) \\
Female sex (vs male) & Vessel treated (M2 vs MI) \\
BMI $\left(\mathrm{kg} / \mathrm{m}^{2}\right)$ & Vessel treated (all others vs MI) \\
Baseline mRS $=0$ (vs $>0)$ & Rescue therapy, all \\
IV-rtPA failed (vs contraindicated) & Rescue therapy, rtPA \\
Myocardial infarction & Rescue therapy, all mechanical \\
Hypertension & Rescue therapy, Merci only \\
Diabetes & Rescue therapy, Penumbra only \\
Hyperlipidemia & Rescue therapy, angioplasty \\
Smoker & Rescue therapy, medical and mechanical \\
Atrial fibrillation & Rescue therapy, only mechanical \\
Previous ischemic stroke & Any rtPA use (IV or IA, baseline or rescue) \\
NIHSS at baseline & Time to treatment (min) \\
ASPECTS at baseline & Time to recanalization (min) \\
Glucose at baseline & Device passes \\
Hyperglycemia ( $>140$ mmol/L) at baseline & Anesthesia (sedation vs general) \\
Systolic BP at baseline & Procedure time (min) \\
Diastolic BP at baseline & TIMI score (core lab) \\
Platelet count at baseline & TICl score (core lab) \\
INR at baseline & TIMI at baseline 1 (vs 0) \\
aPTT at baseline & \\
\hline
\end{tabular}

Note:-INR indicates international normalized ratio; BMI, body mass index; aPTT, activated partial thromboplastin time; BP, blood pressure; TIMI, Thrombolysis In Myocardial Infarction.

Table 2: ICH subtype by group

\begin{tabular}{lccc}
\hline \multicolumn{1}{c}{ ICH Type } & $\begin{array}{c}\text { All Solitaire } \\
(\boldsymbol{n}=\mathbf{8 9})\end{array}$ & $\begin{array}{c}\text { All Merci } \\
(\boldsymbol{n}=\mathbf{5 5})\end{array}$ & $\begin{array}{c}\text { Difference } \\
(\boldsymbol{P} \text { Value) }\end{array}$ \\
\hline All ICH & $21.3 \%(19 / 89)$ & $38.2 \%(21 / 55)$ & .035 \\
Symptomatic & $1.1 \%(1 / 89)$ & $10.9 \%(6 / 55)$ & .012 \\
SAH & $2.2 \%(2 / 89)$ & $12.7 \%(7 / 55)$ & .027 \\
IVH & $1.1 \%(1 / 89)$ & $7.3 \%(4 / 55)$ & .070 \\
PH1 & $4.5 \%(4 / 89)$ & $3.6 \%(2 / 55)$ & 1.00 \\
PH2 & $1.1 \%(1 / 89)$ & $7.3 \%(4 / 55)$ & .070 \\
Basal ganglia & $15.7 \%(14 / 89)$ & $14.5 \%(8 / 55)$ & 1.00 \\
Cortical & $4.5 \%(4 / 89)$ & $14.5 \%(8 / 55)$ & .058 \\
\hline
\end{tabular}

rhage as symptomatic. ${ }^{1,8}$ In addition, other aspects of $\mathrm{ICH}$, including subarachnoid versus intraparenchymal, extent (petechial hemorrhage versus frank hematoma), and topographic location (deep versus superficial) have been identified in some studies as predictors of poor outcome after interventional treatment of acute ischemic stroke. ${ }^{9-11}$ The Solitaire FR With Intention For Thrombectomy (SWIFT; https:/clinicaltrials.gov/ct2/show/ NCT01054560) trial used a refined definition of SICH: any parenchymal hematoma (PH), subarachnoid hemorrhage, or intraventricular hemorrhage (IVH) associated with a worsening of National Institute of Health Stroke Scale score by $\geq 4$ within 24 hours. ${ }^{12}$ Compared with SICH definitions used in earlier intravenous and intra-arterial fibrinolysis trials, this definition incorporated a broader range of hemorrhage types and excluded minor petechial hemorrhages unlikely to be causally related to neurologic worsening. ${ }^{13-17}$

The SWIFT trial demonstrated the superiority of the Solitaire FR stent retriever device (Covidien, Irvine, California) over the Merci coil retriever device (Concentric Medical, Mountain View, California) with respect to revascularization and clinical outcome. Notably, SWIFT also found a substantially lower rate of SICH with stent versus coil retrieval $(2.0 \%$ versus $11 \%$ ).

The aims of this study were to define the procedural and clin- ical factors related to the occurrence of SICH in the SWIFT trial and to determine the ICH features with the greatest impact on clinical outcome.

\section{MATERIALS AND METHODS}

The SWIFT trial methods have been previously described. ${ }^{18}$ The primary efficacy end point of the study was arterial recanalization of the target vessel measured by a Thrombolysis in Myocardial Infarction score of 2 or 3 without SICH after up to 3 passes of the assigned device, as assessed by an independent core laboratory. In patients for whom adequate recanalization was not achieved, rescue therapy was allowed with a different regulatory agency-cleared neurovascular thrombectomy device, intraarterial fibrinolysis with rtPA, or both.

In the present study, ICHs were characterized by compartment (intraparenchymal, subarachnoid, intraventricular) and symptomatic status (asymptomatic and $\mathrm{SICH}$ ). Intraparenchymal hemorrhages were further subdivided by extent (hemorrhagic infarction type 1, hemorrhagic infarction type 2, parenchymal hematoma type 1 , parenchymal hematoma type 2 ) and brain location (cortical versus deep/basal ganglionic). Basal ganglia ICH was determined by vascular distribution and included any hemorrhage within the territory of the lenticulostriate, Heubner, and the anterior choroidal arteries. SAH, IVH, hemorrhagic infarction-1, hemorrhagic infarction-2, $\mathrm{PH}-1, \mathrm{PH}-2$, and basal ganglia $\mathrm{ICH}$ classifications were defined by an independent blinded core laboratory.

The entire study population was analyzed, including patients enrolled in both the roll-in and randomized phases. All analyzed clinical and imaging data except for ASPECTS were collected prospectively as part of the SWIFT main trial protocol. ASPECTSs were obtained by review of the CT imaging data base by 2 physicians (R.J., D.L.) blinded to the assigned device.

In univariate analysis, candidate predictors of ICH included 21 pretreatment demographic, clinical, and imaging variables and 20 procedural variables (Table 1 ). The associations of ICH features with clinical outcomes were analyzed for the following end points: NIHSS (at 24 hours, 7 days/discharge, 30 days, 90 days), Barthel Index of Activities of Daily Living (90 days), modified Rankin Scale of global disability (90 days), trial-defined good neurologic outcome (90 days), and mortality at 90 days. The prespecified tripartite definition of good neurologic outcome in the trial was the following: 1 ) $\mathrm{mRS}$ of $\leq 2$, or 2 ) mRS equal to the prestroke $\mathrm{mRS}$ if the prestroke $\mathrm{mRS}$ was $>2$, or 3 ) NIHSS score improvement of $\geq 10$ points.

\section{Statistical Analysis}

The rate of ICH types was compared between groups by using the Fisher exact test. Univariate logistic models with treatment device included as a nuisance parameter were run on each individual predictor, and predictors with a univariate $P$ value $<.20$ were 
Table 3: Predictors of ICH subtypes

\begin{tabular}{|c|c|c|c|c|c|}
\hline ICH Subtype & Predictor & Odds Ratio & Lower $\mathrm{Cl}$ & Upper Cl & $P$ Value \\
\hline Any ICH & Rescue therapy, IA rtPA & 12.06 & 1.082 & 134.457 & .043 \\
\hline $\mathrm{SICH}$ & NIHSS at baseline & 1.24 & 1.023 & 1.344 & .029 \\
\hline \multirow[t]{2}{*}{$\mathrm{SAH}$} & ASPECTS at baseline & 3.113 & 1.173 & 8.261 & .023 \\
\hline & Rescue therapy, IA rtPA & 12.46 & 1.731 & 89.708 & .012 \\
\hline IVH & Rescue therapy, IA rtPA & 8.846 & 1.184 & 66.079 & .033 \\
\hline $\mathrm{PH} 2$ & INR at baseline & 7.267 & 1.22 & 43.288 & .029 \\
\hline \multirow[t]{3}{*}{ Basal ganglia } & Rescue therapy, IA rtPA & 18.29 & 1.89 & 176.966 & .012 \\
\hline & Time to treatment $(\mathrm{min})^{\mathrm{a}}$ & 1.114 & 1.029 & 1.207 & .008 \\
\hline & TIMI score (core lab) & 1.744 & 1.009 & 3.012 & .046 \\
\hline \multirow[t]{2}{*}{ Cortical } & NIHSS at baseline & 1.19 & 1.015 & 1.278 & .031 \\
\hline & Rescue therapy, IA rtPA & 15.406 & 2.662 & 89.149 & .002 \\
\hline
\end{tabular}

a Time to treatment denotes time from symptoms onset to groin puncture.

Table 4: Difference in ICH predictors between the 2 treatment groups

\begin{tabular}{lccc}
\hline \multicolumn{1}{c}{ ICH Predictors } & $\begin{array}{c}\text { All Solitaire } \\
(\boldsymbol{n}=\mathbf{8 9})\end{array}$ & $\begin{array}{c}\text { All Merci } \\
(\boldsymbol{n}=\mathbf{5 5})\end{array}$ & $\boldsymbol{P}$ Value \\
\hline NIHSS (baseline) (mean) & $17.4 \pm 4.5(89)$ & $17.5 \pm 5.1(55)$ & .88 \\
INR (baseline) (mean) & $1.2 \pm 0.3(88)$ & $1.2 \pm 0.3(54)$ & .97 \\
ASPECTS (baseline) (median) & $8.4 \pm 1.6(86)$ & $8.4 \pm 1.4(53)$ & .98 \\
TIMI 2-3 (final) & $66.7 \%(54 / 81)$ & $30.2 \%(16 / 53)$ & $<.001$ \\
Rescue therapy with IA rtPA & $3.4 \%(3 / 89)$ & $10.9 \%(6 / 55)$ & .085 \\
\hline
\end{tabular}

entered into a multivariable logistic regression model. The backward selection method by using a criterion of $P<.10$ was used to identify the predictors of each ICH type. The difference in the identified independent predictors between groups was tested by using a $t$ test for continuous measures and a Fisher exact test for discrete measures. The effect of ICH type on outcomes was tested by using univariate logistic models with the treatment device included as a nuisance parameter.

\section{RESULTS}

\section{Hemorrhage Frequency by Treatment Group}

As shown in Table 2, among the 144 enrolled patients, any radiologic intracranial hemorrhage (21.3\% versus $38.2 \%)$ and symptomatic intracranial hemorrhage (1.1\% versus $10.9 \%)$ occurred less frequently in the Solitaire FR than Merci retriever arms. Among the hemorrhage compartment and anatomic subtypes, subarachnoid hemorrhage (2.2\% versus $12.7 \%$ ) occurred less frequently with the Solitaire FR than the Merci retriever, with trends also toward less frequent intraventricular hemorrhage, parenchymal hematoma type 2 , and cortical intracerebral hemorrhage.

\section{Predictors of Hemorrhagic Subtypes}

Table 3 shows the independent predictors for all intracranial hemorrhage and 6 hemorrhage subtypes. Among all analyzed clinical, imaging, and procedural parameters for each individual hemorrhage subtype included in the multivariate model, the most consistent predictor was rescue therapy with intra-arterial (IA)$\mathrm{rtPA}$, which was an independent predictor for any intracranial hemorrhage, SICH, SAH, IVH, PH2, and cortical hemorrhage. Other predictors included the following: baseline NIHSS score (for SICH and cortical ICH), baseline ASPECTS (for SAH), baseline international normalized ratio (for $\mathrm{PH} 2$ ), and time to treatment and recanalization (Thrombolysis In Myocardial Infarction 2-3 for basal ganglionic ICH). Among the identified independent predictors of hemorrhage, achievement of recanalization differed strongly between the 2 treatment arms, and the use of rescue IA-rtPA showed a trend toward a difference (Table 4).

\section{Effect on Outcome}

Among all hemorrhagic types analyzed, basal ganglia ICH and SICH had the greatest effect on outcomes, each statistically associated with 4 of 8 outcomes and trending toward association with 3 additional outcomes (Table 5). The basal ganglia $\mathrm{ICH}$ was inversely associated with good neurologic outcome at 90 days (OR, $0.300 ; 95 \% \mathrm{CI}, 0.105-0.860 ; P=.025)$, nondisabled outcome (mRS, $0-2$ ) at 90 days (OR, 0.248 ; $95 \%$ CI, $0.068-0.897$; $P=.034$ ), higher NIHSS scores at 24 hours (OR, 5.772; 95\% CI, 2.209-9.337; $P=.002$ ), and NIHSS score at 7 days/discharge (OR, 8.008; 95\% CI, 2.247-13.545; $P=.005$ ). SICH was associated with mortality at 90 days (OR, 5.734; 95\% CI, 1.012-32.489; $P=.048)$, NIHSS score at 7 days/discharge, (OR, 17.998; 95\% CI, $8.006-27.990 ; P=.001)$, NIHSS score at 90 days (OR, 22.354; 95\% CI, 7.285-37.424; $P=.004$ ), and level of disability (mRS shift) at 90 days (OR, 2.414; 95\% CI, 0.582-4.247; $P=.011$ ). Any intracranial hemorrhage was associated with NIHSS score at 24 hours (OR, 4.856; 95\% CI, 1.964-7.749; $P=.001$ ) and NIHSS score at 7 days/discharge (OR, 6.130; 95\% CI, 1.538-10.722; $P=$ .002). PH1, PH2, SAH, IVH, and cortical ICH were not associated with any of the clinical outcomes.

\section{DISCUSSION}

This study provides important insight into the pathophysiologic mechanisms of different ICH subtypes after mechanical revascularization and their respective impacts on clinical outcome.

Among all clinical and baseline predictors, NIHSS score was the strongest predictor of SICH in this study. However, this association does not explain the lower incidence of hemorrhage in the patients treated with the Solitaire because the baseline NIHSS score did not differ between the 2 groups. It is likely that other procedural factors causing specific hemorrhagic subtypes were contributing to the overall higher rate of $\mathrm{SICH}$ in the Merci group.

The higher incidence of SAH in the Merci group compared with the Solitaire group ( $12.7 \%$ versus $2.2 \% ; P=.027)$ is similar to that in previously reported data. The higher frequency of SAH with coil retrievers has several potential sources, including greater device injury to the vessel wall; greater required traction of the device on clot resulting in displacement of the vascular tree; the need for multiple retriever passes, increasing the likelihood of dissection; and the more frequent need for rescue therapy with IA-rtPA with attendant increased bleeding risk. ${ }^{19}$

As opposed to the Merci device, which is initially delivered distal to the target thrombus, the Solitaire FR expands radially within the thrombus when unsheathed from the microcatheter. This outward expansion through the thrombus allows stable positioning within the vessel wall, which likely minimizes the risk of vessel perforation and/or microdissection during delivery. ${ }^{20}$ In addition, the mechanical properties of the Solitaire allow optimal 


\begin{tabular}{|c|c|c|c|c|c|c|c|c|}
\hline \multirow[b]{2}{*}{ Outcome } & \multicolumn{8}{|c|}{ Hemorrhage Type } \\
\hline & All ICH & BG & $\mathrm{SICH}$ & $\mathrm{PH} 1$ & $\mathrm{PH} 2$ & SAH & IVH & Cortical \\
\hline NIHSS at 24 hours & .001 (OR, 4.85) & $.002(\mathrm{OR}, 5.77)$ & .067 & .329 & .087 & .659 & .988 & .798 \\
\hline NIHSS at 7 days/discharge & .002 (OR, 6.13) & .005 (OR, 8.01) & .001 (OR, 17.99) & .747 & .089 & .386 & .160 & .508 \\
\hline NIHSS at 30 days & .141 & .055 & .068 & 609 & .453 & .795 & .070 & .611 \\
\hline NIHSS at 90 days & .195 & .067 & .004 (OR, 22.35) & .821 & .362 & .725 & .152 & .464 \\
\hline $\mathrm{mRS}$ shift at 90 days & .088 & .061 & .011 (OR, 2.41) & .675 & .431 & .949 & .771 & .453 \\
\hline mRS $0-2$ at 90 days & .057 & $.034(\mathrm{OR}, 0.24)$ & .122 & .899 & .770 & .671 & .770 & .626 \\
\hline Good neuro-outcome at 90 days $^{b}$ & .079 & .025 (OR, 0.30) & .062 & .960 & .528 & .531 & .528 & .271 \\
\hline Mortality at 90 days & .731 & .382 & .048 (OR, 5.73) & .575 & .763 & .994 & .763 & .250 \\
\hline
\end{tabular}

Note:-BG indicates basal ganglia.

${ }^{\text {a }}$ ORs are provided for $P$ values $\leq .05$, which are significant

${ }^{b}$ An $m R S$ of $\leq 2$ or equal to the prestroke mRS if the prestroke mRS was $>2$ or an NIHSS score improvement of $\geq 10$ points.

wall conformance and flexibility and a minimal vessel-straightening effect, likely reducing the risk of endothelial damage during the pull. Finally, the efficient clot engagement and rapid revascularization decrease the need for multiple passes and additional rescue therapy. Although our data did not reveal any association between the number of passes and hemorrhage occurrence, rescue therapy with IA-rtPA strongly correlated with multiple hemorrhagic subtypes, including any intracranial hemorrhage, SICH, $\mathrm{SAH}, \mathrm{IVH}, \mathrm{PH} 2$, and cortical hemorrhage. Any use of rtPA (IV or IA, baseline, or rescue) did not impact hemorrhage occurrence; this outcome suggests that the deleterious effect of the thrombolytic therapy is only present when used intra-arterially as a rescue therapy, after initial mechanical thrombectomy. As such, preinterventional use of IV-rtPA is not discouraged. The relationship between IA-rtPA rescue therapy and subarachnoid hemorrhage was particularly strong, increasing the odds of SAH more than 12 -fold. These findings indicate that the fibrinolytic effect of the medication used as a rescue therapy likely potentiates device-induced vascular injury, eventually resulting in intracranial hemorrhage, particularly SAH.

Of all hemorrhagic subtypes, basal ganglia was the only hemorrhage associated with successful reperfusion. Parenchymal ischemic injury may progress particularly rapidly in subcortical, basal ganglia regions, due to rapidly evolving infarction within the deep subcortical tissue in the presence of occluded ostia of lenticulostriate arteries that lack collateral flow. This more advanced ischemic tissue destruction may render subcortical tissues more vulnerable to reperfusion-mediated hemorrhage. Basal ganglionic hemorrhage has been previously associated with poor outcome after mechanical thrombectomy. ${ }^{10}$ The association of time to treatment with basal ganglia ICH (OR, 1.14; 95\% CI, 1.029$1.207 ; P=.008$ ) in our study demonstrates that for every 10 minute delay, there is an approximate $11 \%$ higher risk of hemorrhage within the basal ganglia (Fig 1). This significant impact on time to treatment confirms the vulnerability of the deep subcortical structures to prolonged ischemia. The distinctive determinants and clinical impact of basal ganglionic-versus-cortical hemorrhage demonstrate the importance of identifying these subtypes, in addition to clinical type (symptomatic versus asymptomatic) and extent (hematoma versus hemorrhagic infarction). Given the strong impact of basal ganglionic hemorrhages on the outcome in our study, it is reasonable to consider this hemorrhagic subtype as an important predictor of outcome in clinical

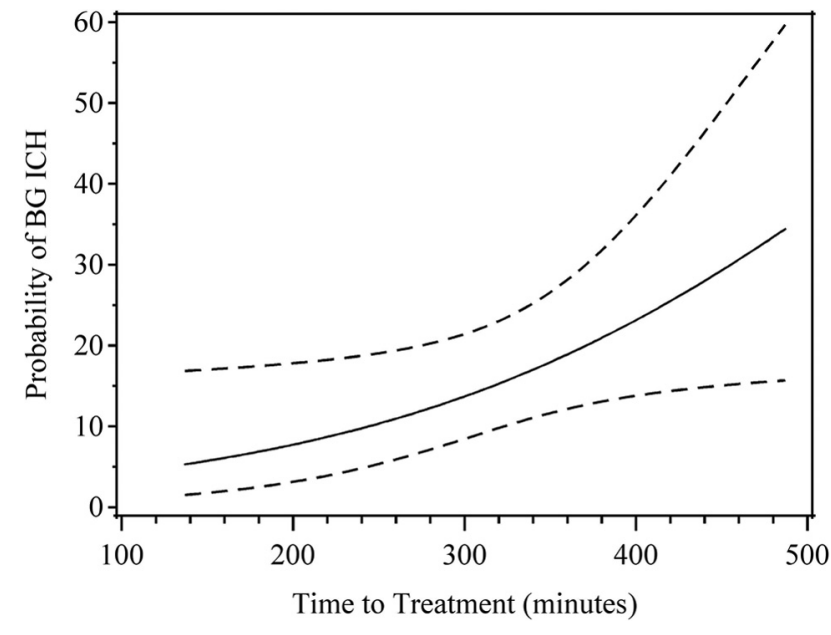

FIG 1. Plot of predicted probability (\%) of basal ganglia ICH based on time to treatment. The dotted lines represent the upper and lower confidence bounds of the predicted probability.

practice and in future studies of endovascular revascularization for acute ischemic stroke.

\section{Limitations}

The main limitation of our study was the relatively small number of hemorrhages in the SWIFT trial, potentially confounding the results of the regression model as demonstrated by the wide confidence intervals in Table 3. This limitation explains the lack of definitive association between $\mathrm{PH} 2$ and clinical outcome (Table 5). In addition, our outcome data were derived on the basis of univariate analysis instead of multivariate regression, focusing only on the hemorrhage effect and excluding other clinical and procedural variables. However, the goal of the outcome analysis was to analyze the clinical effect of each ICH subtype compared with all other hemorrhages, rather than identifying overall predictors of clinical outcome.

\section{CONCLUSIONS}

The higher rate of intracranial hemorrhage observed in the SWIFT trial with the Merci retriever versus the Solitaire FR was mostly related to increased occurrence of SAH and to a lesser degree of IVH, PH2, and cortical ICH. Each one of these ICH subtypes was strongly associated with IA-rtPA rescue therapy. In comparison with the Merci device, the Solitaire likely offsets SICH due to a lower incidence of vascular injury and a decreased need for additional IA rtPA administration. 
Basal ganglia ICH has a substantial deleterious effect on clinical outcome and is related in part to reperfusion of subcortical tissues that have already sustained advanced ischemic injury, independent of the type of device used.

These findings warrant cautious use of additional rescue IArtPA after mechanical thrombectomy, particularly in patients with established basal ganglia infarcts and prolonged time from symptom onset.

Disclosures: Reza Jahan—RELATED: Covidien*; Consulting Fee or Honorarium: Covidien; Support for Travel to Meetings for the Study or Other Purposes: Covidien; UNRELATED: Consultancy: Covidien; Payment for Lectures (including service on speakers bureaus): Covidien; OTHER: Covidien (consultant; member of executive steering committee for SWIFT PRIME; International Neurointerventional Advisor SWIFT PRIME; member of steering committee for the Systemic Evaluation of Patients Treated with Neurothrombectomy Devices for Acute Ischemic Stroke [STARTIS] registry), Medina Medical (Chief Scientific Advisor), Stryker Neurovascular (Speakers Bureau). David Liebeskind-UNRELATED: Consultancy: Stryker Neurovascular, ${ }^{*}$ Covidien*; Payment for Lectures (including service on Speakers Bureaus): Stryker Neurovascular,* Covidien.* Wayne Clark-UNRELATED: Grants/Grants Pending: Oregon Health and Science University. * Raul G. Nogueira-UNRELATED: Fees for Participation in Review Activities such as Data Monitoring Boards, Statistical Analysis, End Point Committees, and the Like: Stryker Neurovascular, Covidien, Penumbra, Comments: Stryker Neurovascular (Thrombectomy Revascularization of Large Vessel Occlusions in Acute Ischemic Stroke-2 and Trevo and Medical Management Versus Medical Management Alone in Wake up and Late Presenting Strokes [DAWN] Trials Principal Investigator; both modest); Covidien (SWIFT and SWIFT PRIME trials steering committee; both modest; Study of Tamoxifen and Raloxifene Trial Angiographic Core Lab; significant); Penumbra (3D Separator Trial Executive Committee; no payment); Editor-In-Chief of Interventional Neurology Journal (no payment); OTHER: CoAxia (clinical trials steering committee). Jeffrey SaverRELATED: Grant: ev3/Covidien*; Consulting Fee or Honorarium: ev3/Covidien*; UNRELATED: Consultancy: Covidien, * Stryker Neurovascular*; OTHER: St. Jude Medical Clinical Trial Steering Committee, ev3 Clinical Trial Steering Committee, CoAxia Clinical Trial Steering Committee, Grifols Clinical Trial Steering Committee, Genervon Clinical Trial Steering Committee, BrainsGate Clinical Trial Steering Committee, Stryker Neurovascular Clinical Trial Steering Committee. ${ }^{\star}$ Money paid to the institution.

\section{REFERENCES}

1. National Institute of Neurological Disorders and Stroke rt-PA Stroke Study Group. Tissue plasminogen activator for acute ischemic stroke. N Engl J Med 1995;333:1581-87 CrossRef Medline

2. Hallevi H, Barreto AD, Liebeskind DS, et al. The HIAT score: identifying patients at high risk for poor outcome after intra arterial therapy for acute ischemic stroke. Stroke 2009;40:E164 CrossRef

3. Flint AC, Faigeles BS, Cullen SP, et al; VISTA Collaboration. THRIVE score predicts ischemic stroke outcomes and thrombolytic hemorrhage risk in VISTA. Stroke 2013;44:3365-69 CrossRef Medline

4. Christoforidis GA, Karakasis C, Mohammad Y, et al. Predictors of hemorrhage following intra-arterial thrombolysis for acute ischemic stroke: the role of pial collateral formation. AJNR Am J Neuroradiol 2009;30:165-70 CrossRef Medline

5. Bang OY, Buck BH, Saver JL, et al. Prediction of hemorrhagic transformation after recanalization therapy using $\mathrm{T} 2{ }^{\star}$-permeability magnetic resonance imaging. Ann Neurol 2007;62:170-76 CrossRef Medline

6. Khatri P, Broderick JP, Khoury JC, et al; IMS I and II Investigators. Microcatheter contrast injections during intra-arterial thromboly- sis may increase intracranial hemorrhage risk. Stroke 2008;39: 3283-87 CrossRef Medline

7. Brekenfeld C, Remonda L, Nedeltchev K, et al. Symptomatic intracranial haemorrhage after intra-arterial thrombolysis in acute ischaemic stroke: assessment of 294 patients treated with urokinase. J Neurol Neurosurg Psychiatry 2007;78:280-85 Medline

8. Saver JL. Hemorrhage after thrombolytic therapy for stroke: the clinically relevant number needed to harm. Stroke 2007;38:2279-83 CrossRef Medline

9. Larrue V, von Kummer RR, Müller A, et al. Risk factors for severe hemorrhagic transformation in ischemic stroke patients treated with recombinant tissue plasminogen activator: a secondary analysis of the European-Australasian Acute Stroke Study (ECASS II). Stroke 2001;32:438-41 CrossRef Medline

10. Loh Y, Towfighi A, Liebeskind DS, et al. Basal ganglionic infarction before mechanical thrombectomy predicts poor outcome. Stroke 2009;40:3315-20 CrossRef Medline

11. Wahlgren N, Ahmed N, Dávalos A, et al; SITS-MOST investigators. Thrombolysis with alteplase for acute ischaemic stroke in the Safe Implementation of Thrombolysis in Stroke-Monitoring Study (SITS-MOST): an observational study. Lancet 2007;369:275-82 CrossRef Medline

12. Saver JL, Jahan R, Levy EI, et al; SWIFT Trialists. Solitaire flow restoration device versus the Merci retriever in patients with acute ischaemic stroke (SWIFT): a randomised, parallel-group, non-inferiority trial. Lancet 2012;380:1241-49 CrossRef Medline

13. Gobin YP, Starkman S, Duckwiler GR, et al. MERCI 1: a phase 1 study of mechanical embolus removal in cerebral ischemia. Stroke 2004; 35:2848-54 CrossRef Medline

14. Smith WS, Sung G, Starkman S, et al; MERCI Trial Investigators. Safety and efficacy of mechanical embolectomy in acute ischemic stroke: results of the MERCI trial. Stroke 2005;36:1432-38 CrossRef Medline

15. Smith WS, Sung G, Saver J, et al; Multi MERCI Investigators. Mechanical thrombectomy for acute ischemic stroke: final results of the Multi MERCI trial. Stroke 2008;39:1205-12 CrossRef Medline

16. Penumbra Pivotal Stroke Trial Investigators. The Penumbra pivotal stroke trial: safety and effectiveness of a new generation of mechanical devices for clot removal in intracranial large vessel occlusive disease. Stroke 2009;40:2761-68 CrossRef Medline

17. Furlan A, Higashida R, Wechsler L, et al; PROACT Investigators. Intra-arterial prourokinase for acute ischemic stroke: the PROACT II study—a randomized controlled trial. JAMA 1999;282:2003-11 CrossRef Medline

18. Saver JL, Jahan R, Levy EI, et al; SWIFT Trialists. SOLITAIRE ${ }^{\mathrm{TM}}$ with the intention for thrombectomy (SWIFT) trial: design of a randomized, controlled, multicenter study comparing the SOLITAIRE ${ }^{\mathrm{TM}}$ Flow Restoration device and the MERCI Retriever in acute ischaemic stroke. Int J Stroke 2014;9:658-68 CrossRef Medline

19. Shi ZS, Liebeskind DS, Loh Y, et al; UCLA Endovascular Stroke Therapy Investigators. Predictors of subarachnoid hemorrhage in acute ischemic stroke with endovascular therapy. Stroke 2010;41:2775-81 CrossRef Medline

20. Akins PT, Amar AP, Pakbaz RS, et al; SWIFT Investigators. Complications of endovascular treatment for acute stroke in the SWIFT trial with Solitaire and Merci devices. AJNR Am J Neuroradiol 2014; 35:524-28 CrossRef Medline 